\title{
DESENVOLVIMENTO DE UM PLANO DE AÇÃO PARA O ENSINO SUPERIOR A PARTIR DE FERRAMENTAS DE GESTÃO ESTRATÉGICA E MODELOS DE NEGÓCIOS PARA EQUIPES
}

\author{
DESENVOLVIMIENTO DE UN PLAN DE ACCIÓN PARA LA ENSEÑANZA \\ UNIVERSITARIA A PARTIR DE HERRAMIENTAS DE PLANIFICACIÓN \\ ESTRATÉGICO Y MODELOS DE NEGOCIACIÓN PARA EQUIPOS
}

DEVELOPMENT OF AN ACTION PLAN FOR HIGHER EDUCATION USING
STRATEGIC MANAGEMENT TOOLS AND TEAM BUSINESS MODELS

Cristiane Regina Ruiz ${ }^{1}$

RESUMO: As mudanças no cenário da educação com a atualização dos instrumentos de avaliação do MEC em 2017 mostram que a mentalidade das IES (Instituições de Ensino Superior) precisa ser renovada com foco maior em planejamento, inovação e disrupção. O papel do coordenador de curso deve estar pautado em um plano de ação documentado e compartilhado, administrando a potencialidade do corpo docente e favorecendo a integração e a melhoria contínua. A gestão estratégica auxilia na ruptura de antigos paradigmas e na implementação de ideias inovadoras. Diante desses novos desafios o Centro Universitário São Camilo - SP propôs, por meio deste artigo, relatar sua experiência no que diz respeito ao processo de construção conjunta entre docentes e coordenação para a criação de um plano de ação anual. A criação e implementação deste plano de ação tiveram um impacto positivo na condução do planejamento acadêmico, além de auxiliar na detecção precoce de problemas, no comprometimento do gestor e da equipe, na identificação da necessidade de redefinições de ações, além de estimular uma abordagem cooperativa e ajudar a ordenar ações individuais para esforço global.

PALAVRAS-CHAVE: Planejamento. Plano de ação. Gestão estratégica. Graduação. Educação. Ensino superior.

RESUMEN: Los cambios en el escenario de la educación con la actualización de los instrumentos de evaluación del MEC en 2017 ponen en evidencia que la mentalidad de las IEs necesita ser renovada con foco mayor en planificación, innovación y disrupción. El rol del coordinador del curso debe estar centrado en un plan de acción documentado y compartido administrando la potencialidad del cuerpo docente y favoreciendo la integración y la mejoría continua. La gestión estratégica auxilia en la ruptura de antiguos paradigmas y en la implementación de ideas innovadoras. Delante de esos nuevos desafios, el Centro Universitario São Camilo - SP propuso por medio de este artículo relatar su experiencia en lo que dice respeto al proceso de construcción conjunta entre docentes y coordinación para creación de un plan de acción anual. La creación y la implementación de este plan de acción tuvieron un efecto positivo en la conducción de la planificación académica además de auxiliar en la

${ }^{1}$ Centro Universitário São Camilo, São Paulo - SP - Brasil. Doutora em Ciências (Morfologia) pela Escola Paulista de Medicina (UNIFESP/ EPM). Coordenadora dos Eixos Institucionais do Centro Universitário São Camilo. ORCID: https://orcid.org/0000-0001-7140-8220. E-mail: crisruiz@ saocamilo-sp.br 
detección precoz de problemas, en el compromiso del gestor y del equipo, en la identificación de la necesidad de redefinición de acciones, además de estimular un abordaje cooperativo y ayudar a ordenar acciones individuales para esfuerzo global.

PALABRAS CLAVE: Planificación. Plan de acción. Gestión estratégica. Licenciatura. Educación. Enseñanza universitaria.

ABSTRACT: Changes in the education scenario due to MEC's (Ministry of Education) evaluation instruments update in 2017 show that HEI (Higher Education Institutions) mentality needs to be renewed with a bigger focus on planning, innovating and disrupting. The course coordinator's role must be guided by a documented and shared action plan, administrating the faculty's potential and supporting continual improvement and integration. Strategic management assists in breaking old paradigms and implementing innovative ideas. In light of these new challenges the São Camilo University Center - SP proposed through this article, to report its experience concerning the joint construction process between teachers and coordinators to create an annual action plan. The creation and implementation of this action plan had a positive impact in academic planning conduction, besides assisting in the early detection of problems, team and manager's commitment, identifying an action renewal need, in addition to stimulating a cooperative approach and helping to command individual actions focusing on a global effort.

KEYWORDS: Planning. Action plan. Strategic management. Undergraduate course. Education. Higher education.

\section{Introdução}

De acordo com as mudanças significativas na legislação do ensino superior e nos indicadores de avaliação descritos no novo instrumento de avaliação dos cursos de graduação, publicado em 2017, as IES perceberam a necessidade de atualizar suas estratégias visto que inúmeros conceitos ganharam importância no novo instrumento, como o planejamento, que foi citado 4 vezes no instrumento de avaliação de 2015 e citado 28 vezes no instrumento de 2017, no que diz respeito ao Reconhecimento de cursos de graduação. Do mesmo modo, o conceito Inovação passa de nenhuma citação em 2015 para 16 citações em 2017 (REIS; COVAC; COVAC, 2017).

A atuação do coordenador de curso, na visão do novo instrumento, deve estar pautada em um plano de ação documentado e compartilhado, administrando a potencialidade do corpo docente e favorecendo a integração e a melhoria contínua (INEP, 2017).

No processo de inovação e criação na esfera educacional, contamos com os momentos de planejamento acadêmico, espaço propício para a troca de ideias e experiências, reflexão sobre o que foi realizado no período letivo anterior e momento de elencar as prioridades para o 
próximo período letivo. Atualmente, o coordenador gestor necessita, mais do que nunca, exercitar uma liderança participativa, alinhando propostas de mudança com sua equipe, sendo objetivo, transparente e motivador, visto que a partir do momento em que a equipe se mostra comprometida obtemos a visão compartilhada do processo, fator imprescindível para alcançar o compartilhamento das responsabilidades e a tomada de decisão partida de uma postura de cooperação (VAN DER PIJL; LOKITZ; SOLOMON, 2018).

Para alcançar melhores resultados na gestão de equipes, o planejamento estratégico demonstra ser um modelo reflexivo que exige a aquisição e gestão de informação, o que demanda considerável investimento de diferentes recursos institucionais. Caggy e Benevides (2018) nos alertam que a gestão estratégica é um processo contínuo e interativo que visa manter uma organização como um conjunto apropriadamente integrado a seu ambiente. Ao aplicarmos essa premissa a um grupo de aproximadamente 40 docentes, os maiores desafios são o tempo e a diversidade da equipe.

O coordenador enquanto gestor conduz as ações de seus docentes e define iniciativas de implantação de novos modelos de trabalho, ferramentas de análise e monitora o que foi realizado. O plano de ação, ao ser usado no âmbito educacional, tem condições de traduzir uma estratégia em termos operacionais, alinhar a equipe à estratégia proposta e transformá-la em tarefa de todos, ao mesmo tempo que a converte em processo e mobiliza a mudança de cenário por meio da liderança executiva (CAGGY; BENEVIDES, 2018; VAN DER PIJL; LOKITZ; SOLOMON, 2018).

O objetivo deste trabalho é relatar a experiência do Centro Universitário São Camilo SP, no que diz respeito ao processo de construção conjunta dos docentes com a coordenação dos Eixos Institucionais na criação de um plano de ação anual com vigência de 2019/1 a 2019/2.

A fase anterior à geração de um plano de ação ocorreu em 2018/2, durante o XXIII Workshop de Integração Docente, com a criação de um modelo Canvas para planejamento acadêmico aliado a ferramentas de Design Thinking (RUIZ, 2019), o que permitiu a seleção de títulos de disciplinas e ementas a serem revitalizados, a aproximação de disciplinas afins com integração de conteúdos e a atualização das metodologias de ensino e de avaliação, resultando em uma mudança positiva essencial na equipe de docentes. Como sempre estamos em busca de aprimoramentos que gerem resultados, essa primeira etapa foi crucial para que ocorresse uma mudança de modelo mental nos docentes em relação à concepção de um plano de ensino e do que é trabalho em equipe.

O próximo passo aconteceu em 2019/1 a partir do XXIV Workshop de Integração Docente, realizado semestralmente pelo Centro Universitário São Camilo - SP. Nosso objetivo 
foi trabalhar com a equipe docente o conceito de inovação em relação à gestão de cada uma de suas disciplinas, a partir da criação de um plano de ação para o ano de 2019 que deveria ser implantado, revisto e remodelado quantas vezes fosse necessário durante sua vigência para propiciar flexibilidade, integração e acima de tudo a melhoria da qualidade de cada disciplina.

Esta experiência foi vivenciada pelos docentes dos Eixos Institucionais, parte integrante da estrutura acadêmica institucional que trabalha com alunos de diferentes cursos da instituição (Psicologia, Nutrição, Enfermagem, Fisioterapia, Administração e Tecnologia em Radiologia) do primeiro ao quarto semestre nos quais os discentes estudam disciplinas básicas em salas mistas.

\section{Relato da experiência}

O objetivo principal das atividades foi a integração entre os docentes, por meio do uso de ferramentas de gestão estratégica e modelos de negócios para equipes, no intuito de construir um plano de ação pautado na experiência pessoal e profissional de cada um, dividindo os planos de ação em 4 vertentes (Quadro 1): disciplinas do eixo de ciências biológicas, disciplinas do eixo de ciências exatas, disciplinas do eixo de ciências humanas e disciplinas ministradas em EaD.

Quadro 1 - Disciplinas pertencentes aos Eixos Institucionais

\begin{tabular}{|l|}
\hline Disciplinas do eixo de ciências biológicas \\
\hline Biologia celular e histologia \\
\hline Anatomia humana \\
\hline Bioquímica \\
\hline Neurofisiologia \\
\hline Parasitologia \\
\hline Microbiologia \\
\hline Patologia \\
\hline Disciplinas do eixo de ciências exatas \\
\hline Métodos quantitativos e raciocínio matemático \\
\hline Epidemiologia \\
\hline Disciplinas do eixo de ciências humanas \\
\hline Leitura e Produção de Texto Científico \\
\hline Subjetividade e interações profissionais \\
\hline $\begin{array}{l}\text { Sociedade contemporânea: relações de trabalho e de } \\
\text { consumo no contexto urbano }\end{array}$ \\
\hline $\begin{array}{l}\text { Multiculturalismo, diferenças e diversidade na } \\
\text { perspectiva dos direitos humanos }\end{array}$ \\
\hline Disciplinas ministradas em ead \\
\hline Linguagem e comunicação \\
\hline Bioestatística \\
\hline Modelos de atenção à saúde \\
\hline Políticas públicas e saúde no Brasil \\
\hline Metodologia da pesquisa científica \\
\hline Gestão de serviços e empreendedorismo \\
\hline Bioética \\
\hline
\end{tabular}

Fonte: Elaborado pela autora 
Caggy e Benevides (2018) citam que o conhecimento e a ação compartilhada criam engajamento e comprometimento e, portanto, planejamentos colaborativos são compartilhados, validados e mais efetivos. Deste modo, utilizamos as reuniões pedagógicas como oficinas de criação, dividindo os docentes em grupos por disciplinas e oferecendo a cada grupo um formulário PINT/SIRP (CLARK; HAZEN; PIGNEUR, 2018), no qual, a partir de sua experiência profissional no último semestre e da análise do que foi realizado em seu plano de disciplina, cada grupo deveria discutir e avaliar o que deveria ser colocado no formulário em cada item (Figura 1).

\section{Fase 1 - Ideação}

A intenção do formulário era, num primeiro momento (lado esquerdo do formulário), a partir de uma discussão inicial que colocava o aluno como nosso foco e o plano de ensino do semestre anterior como memória, elencar os problemas em potencial, as principais indagações que o grupo tinha a respeito desses problemas, as necessidades do grupo, exemplificando o que faltou no processo ou o que poderia ser feito de novo ou diferente e as tendências, ou seja, as alternativas para a solução do problema em questão.

Num segundo momento, depois da identificação dos quatro pontos iniciais, a equipe deveria responder (lado direito do formulário), com propostas de valor em potencial, quais seriam as soluções ou sugestões para a resolução do problema, no item inovação, propor novas ideias e estratégias, adaptando-se de modo proativo aos fatos descritos, no segmento recursos, descrever materiais, propriedade intelectual, insumos, entre outras coisas que satisfaçam as necessidades apontadas e, no espaço posicionamento de ideias, explanar como viabilizar as alternativas, maximizando a qualidade e minimizando os riscos ou erros.

As principais características dos formulários que impactaram positivamente os resultados foram: o aspecto visual, que possibilitou a análise e a tomada de decisão dos grupos baseadas em uma única imagem; o aspecto colaborativo, no qual envolvemos equipes mistas que estavam abertas para ouvir, aceitar opiniões diferentes e eram flexíveis a mudanças; o caráter investigativo e experimental, que proporcionou aos docentes a oportunidade de buscar evidências relevantes para agregar valor ao formulário, além de exporem suas próprias ideias; e a simplicidade pois, em uma única atividade, foi possível simplificar o complexo, gerando um material viável e aplicável. 
Figura 1 - Formulário PINT/SIRP aplicado aos docentes
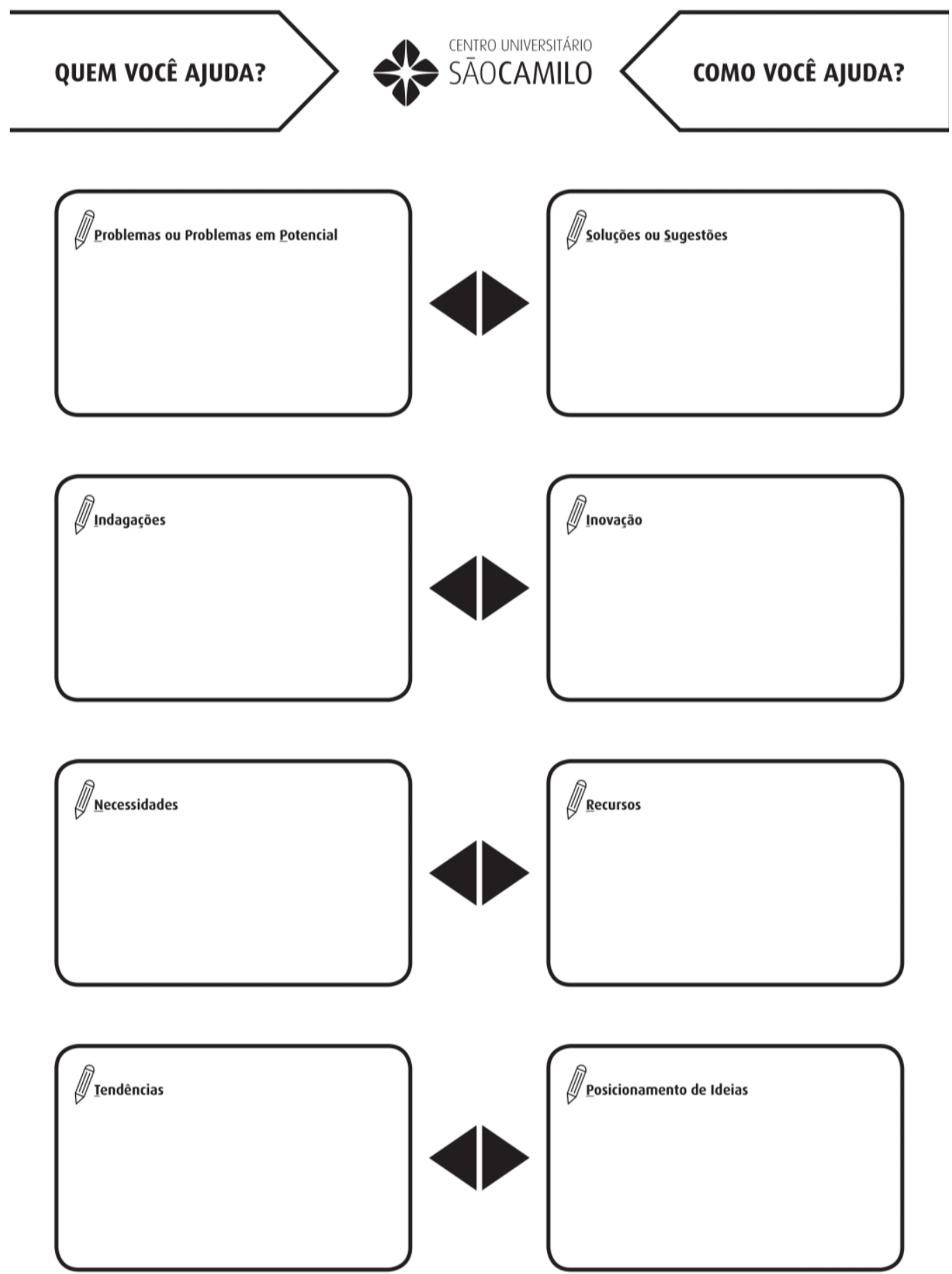

Fonte: Elaborado pela autora

\section{Fase 2 - Planejamento}

A partir dos formulários preenchidos, foi criado um plano de ação para cada eixo e um plano de ação separado para as disciplinas ministradas em EaD. Os planos de ação foram delineados com base na metodologia $5 \mathrm{~W} 2 \mathrm{H}$, porém, adaptados às necessidades institucionais. 
Os itens elencados em cada coluna foram: Ações previstas (o que será feito - What); Justificativa (por que será feito - Why); Plano de execução (como será feito - How); Recursos necessários (aqui decidimos por uma variação do "quanto vai custar - How much") - visto que o plano tem mais cunho acadêmico do que administrativo inserimos informações imprescindíveis sobre o que seria necessário para realizar a ação e atingir a meta -; Responsável (por quem será feito - Who); Cronograma (quando será feito - When).

A estrutura final de cada plano foi definida a partir do conteúdo de cada formulário, dos pontos mais discutidos e das necessidades mais urgentes. O trabalho de compilação dos dados foi realizado pela coordenação dos eixos institucionais e validado pelos docentes. O produto final foi disponibilizado em rede e enviado à reitoria da instituição.

Cada plano de ação descreveu de uma a cinco metas a serem alcançadas no ano de 2019, a saber:

\section{Metas da gestão acadêmica e dos docentes - eixo de exatas}

Meta 1 - Ações para a melhoria do conteúdo programático e desenvolvimento das aulas.

\section{Metas da gestão acadêmica e dos docentes - eixo de ciências biológicas}

Meta 1 - Ações para a melhoria do conteúdo programático;

Meta 2 - Análise das necessidades de aula prática;

Meta 3 - Ações para a melhoria das metodologias de ensino e avaliação.

\section{Metas da gestão acadêmica e dos docentes - eixo de humanas}

Meta 1 - Ações para a melhoria do conteúdo programático;

Meta 2 - Ações para a melhoria das metodologias de ensino e avaliação.

\section{Metas da gestão acadêmica e dos docentes para as disciplinas ministradas em EaD}

Meta 1 - Revisão de materiais e conteúdo de aulas;

Meta 2 - Tutores - definição de papéis e funções;

Meta 3 - Melhoria da qualidade da relação do aluno com as disciplinas em EaD;

Meta 4 - AVA melhorias a serem implantadas;

Meta 5 - Melhoria no âmbito operacional das disciplinas ministradas em EaD.

\section{Fase 3 - Execução e Monitoramento}

Durante o primeiro semestre de 2019 foram realizadas diversas reuniões com os setores envolvidos em cada ação planejada e tivemos inúmeros avanços. No segundo bimestre de 2019/1 realizamos novas reuniões por disciplina, para avaliar o que havia sido cumprido até o momento (sinalizado no plano em azul), o que não havia sido cumprido (sinalizado no plano 
em vermelho) e o que estava em andamento, porém necessitava de maior atenção (sinalizado no plano em amarelo) (Figura 2). Deste modo pudemos evidenciar quais as prioridades para 2019/2 e, também, se algum ponto do plano deve ser abandonado em detrimento de novas necessidades.

Figura 2 - Modelo do plano de ação em andamento

\begin{tabular}{|c|c|c|c|c|}
\hline & & & $\begin{array}{l}\text { que permitam maior } \\
\text { diversificação de } \\
\text { conteúdo }\end{array}$ & \\
\hline \multicolumn{5}{|c|}{ Meta 2 - Tutores - definição de papéis e funções } \\
\hline Ações previstas & Justificativa & Plano de execução & Recursos necessários & Responsável \\
\hline $\begin{array}{l}\text { 1- Definição } \\
\text { documental do } \\
\text { papel dos tutores e } \\
\text { suas funções }\end{array}$ & $\begin{array}{l}\text { Para que os docentes possam } \\
\text { otimizar as atividades dos } \\
\text { tutores é necessário saber } \\
\text { especificamente os limites da } \\
\text { função exercida }\end{array}$ & $\begin{array}{l}\text { Solicitar ao Núcleo de EaD } \\
\text { um documento que } \\
\text { demonstre essas definições }\end{array}$ & $\begin{array}{l}\text { Criação do documento } \\
\text { pelo Nucleo de EaD }\end{array}$ & Núcleo de EaD \\
\hline $\begin{array}{l}\text { 2- Otimização do } \\
\text { trabalho dos } \\
\text { tutores }\end{array}$ & $\begin{array}{l}\text { Os docentes precisam delinear } \\
\text { as atividades juntamente com } \\
\text { os tutores, criando um } \\
\text { envolvimento maior no } \\
\text { acompanhamento efetivo dos } \\
\text { alunos, estimulando a pró- } \\
\text { atividade }\end{array}$ & $\begin{array}{l}\text { Reuniôes entre docentes e } \\
\text { tutores para coloca-los a } \\
\text { par das necessidades das } \\
\text { disciplinas }\end{array}$ & $\begin{array}{l}\text { Uma reunião mensal } \\
\text { presencial ou por } \\
\text { Skype }\end{array}$ & Docentes do EaD \\
\hline $\begin{array}{l}\text { 3- Valorização dos } \\
\text { tutores }\end{array}$ & $\begin{array}{l}\text { Os tutores demonstram } \\
\text { interesse em realizar atividades } \\
\text { de capacitação/ } \\
\text { instrumentalização ofertadas } \\
\text { nola inctitıirãn }\end{array}$ & $\begin{array}{l}\text { Oferecer capacitação } \\
\text { Sway/Forms para os tutores } \\
\text { em dias e horários a } \\
\text { combinar }\end{array}$ & $\begin{array}{l}\text { TI, laboratório de } \\
\text { informática }\end{array}$ & $\begin{array}{l}\text { Coordenação dos } \\
\text { eixos }\end{array}$ \\
\hline
\end{tabular}

Fonte: Elaborado pela autora

\section{Fase 4 - Encerramento}

No início de 2019/2 agendamos o XXV Workshop de Integração Docente para que pudéssemos voltar à análise do plano de ação, revisitando cada item, na tentativa de colocar em prática os que ainda não haviam sido cumpridos ou realizar alterações de acordo com as necessidades emergentes do semestre. A intenção é, ao final de 2019, realizar uma checagem, mediante à utilização do ciclo PDCA, para averiguar cada item que foi planejado, avaliar as ações concluídas, identificar o que foi planejado e o que realmente foi realizado e atuar na correção ou alteração de itens mediante as diferenças identificadas entre planejado/realizado, podendo então caminhar para a conclusão dos planos e dar início a um novo planejamento.

\section{Considerações finais}

Podemos afirmar que a criação e a implementação de um plano de ação construído coletivamente, a partir de um modelo de gestão estratégica, teve um impacto positivo na condução do planejamento acadêmico e também na validação das atividades realizadas e/ou 
não realizadas ao final do período letivo. Neste período de 6 meses, desde sua implantação, percebemos que a construção desse documento auxiliou tanto à gestão quanto aos docentes na detecção precoce de problemas, no comprometimento do gestor e da equipe, na identificação da necessidade de algumas redefinições de ações, além de estimular a abordagem cooperativa, canalizar os esforços para os objetivos definidos em grupo e ajudar a ordenar ações individuais para esforço global.

\section{REFERENCIAS}

CAGGY, R. C.; BENEVIDES, T. M. Strategic canvas: conduza a estratégia do seu negócio por caminhos dinâmicos e criativos de forma inovadora. Rio de Janeiro: Alta Books, 2018.

CLARK, T.; HAZEN, B.; PIGNEUR, Y. Business models for teams: descubra como sua organização realmente funciona e como as pessoas se encaixam nela. Rio de Janeiro: Alta Books, 2018.

INEP, Instituto Nacional de Estudos e Pesquisas Educacionais Anísio Teixeira. Instrumento de avaliação de cursos de graduação presencial e a distância. Brasília: Inep/MEC Instituto Nacional de Estudos e Pesquisas Educacionais Anísio Teixeira, 2017.

REIS, F. J. G.; COVAC, F.; COVAC, J. R. Novos instrumentos de avaliação favorecem inovações nas IES. São Paulo: SEMESP 2017.

RUIZ, C. R. Criação de um modelo Canvas para planejamento acadêmico aliado a ferramentas de Design Thinking. Revista on line de Política e Gestão Educacional, Araraquara, v. 23, n. 2, p. 321-327, maio/ago. 2019. E-ISSN:1519-9029. DOI: https://doi.org/10.22633/rpge.v23i2.11762.

VAN DER PIJL, P.; LOKITZ, J.; SOLOMON, L. K. Planeje melhor seu negócio: novas ferramentas, habilidades e mentalidade para estratégia e inovação. Rio de Janeiro: Alta Books, 2018.

\section{Como referenciar este artigo}

RUIZ, Cristiane Regina. Desenvolvimento de um plano de ação para o ensino superior a partir de ferramentas de Gestão Estratégica e Modelos de Negócios para equipes. Revista on line de Política e Gestão Educacional, Araraquara, v. 24, n. 1, p. 285-293, jan./abr., 2020. e-ISSN:1519-9029. DOI: https://doi.org/10.22633/rpge.v24i1.12909

Submetido em: 09/08/2019

Revisões requeridas: 30/10/2019

Aprovado em: 05/12/2019

Publicado em: 06/01/2020 